\title{
Abdominal adhesions: Surgical treatment and prevention (literature review)
}

\author{
D. A. Kluiko, V.E.Korik, A.N. Sivets \\ Belarusian State Medical University, \\ 83, pr. Dzerzhinskogo, Minsk, 220116, Republic of Belarus
}

For citation: Kluiko D. A., Korik V.E., Sivets A.N. Abdominal adhesions: Surgical treatment and prevention (literature review). Vestnik of Saint Petersburg University. Medicine, 2021, vol. 16, issue 1, pp. 43-49. https://doi.org/10.21638/spbu11.2021.105

The ongoing discussion on the diagnosis and tactics of surgical treatment of painful forms of adhesions of the abdominal cavity and adhesive intestinal obstruction testifies to the persistent urgency of this problem. The presence of a postoperative scar and recurrent abdominal pain are interpreted by surgeons and gastroenterologists as adhesive disease, with the appointment of the same type of treatment. At the same time, patients have a reduced critical attitude to their condition, which leads them to late seeking medical help. For the occurrence of the adhesion process, a long-term effect of a complex of factors is necessary, an important place among which is blood supply, oxygen access to tissues and peristalsis in the early postoperative period. The most effective methods of treatment and prevention of intestinal obstruction are low-traumatic surgical technologies. There was no statistically significant difference between open and laparoscopic adhesiolysis in the number of intraoperative intestinal injuries, wound infections and mortality. Laparoscopic operations have fewer general and pulmonary complications than open ones. Reducing fibroblast proliferation, minimizing hypoxia and inflammation are a new preventive solution to the problem of adhesion formation.

Keywords: adhesive disease of the abdominal cavity, diagnosis, treatment, laparoscopy, adhesiolysis.

Currently, the problem of peritoneal commissures and adhesive intestinal obstruction $(\mathrm{IO})$ is extremely actual $[1 ; 2]$. Despite significant development of medical science, diagnostic, treating and preventive methods of this disease remain debatable. High level of lethality, attaining $27 \%$, work decrement for over than $18 \%$ of patients after surgical intervention, economic expenditures on treating require intensification in search of solution for this scientific-practical problem [3].

There are many empirically accepted rules in approaches for treating peritoneal commissures and its complications, often without sufficient evidence base. Soviet physician A. P.Zilber considered: "Just physicians make a lot of that is considered so since the dawn of time and that was done unreasonably and tritely by our fathers and grandfathers, not considered it necessary to comprehend and to check reasonability and validity of these notorious actions" [4].

Peritoneal commissures implies pain syndrome, intestinal obstruction, dyspepsia due to interintestinal adhesions, caused by serous coat of gastrointestinal wall damage. The cause of peritoneal commissures in $88 \%$ of patients is preceding operations, by $5 \%-$ refer to trauma and inflammatory process in abdominal viscera (AV), $2 \%-$ to congenital anomalies [5].

(C) St. Petersburg State University, 2021 
Pain syndrome intensity in previously operated patients is determined by nervous system condition (threshold of pain sensitivity, pathology of vegetative nervous system etc.), and also by degree of adhesion process, its localization, appearance and commissures density [6]. Preferable therapeutic approach of such patients until recent time had only conservative direction.

Surgeon's arsenal at this moment contains a broad spectrum of peritoneal commissures prevention methods, each of the authors claims that his method enable to decrease probability of adhesion process development, but therewith considerable achievements for last decades didn't happen. Attempts are known to substantiate reasonability of Novocain injection into abdominal cavity, glucocorticoids, thrombolytics, prolonged ferments, 5-fluorouracil, sandostatin and a lot of other preparations. It is possible to suppose, that unsuccessfulness of prevention measures is hidden in insufficient understanding etiology and pathogenesis of abdominale membrane adhesions development [7]. It is well known, that instead of mesotheliocytes fibroblasts are formed, which combine into coarse connective tissue. This process is supported by prolong inflammation.

Commissures formation is undoubtedly associated with mesothelium damage and therewith equally impact on peritoneum causes in part of patients formation of disseminated adhesion process, while other patients undergo complete mesothelium recovery. Experimental investigations demonstrated that animals even of the same line may not respond on simulative factor, due to absence of predisposition to commissures formation. But there is no till now convincing data, determining pathogenetic preconditions of presented observations and general theory of peritoneal commissures pathogenesis has generalized and uncoupled form requiring additional study [8].

For adhesion process appearance prolong impact of multiple factors is required, important place among which is occupied by vascular supply, as well as oxygen access to tissues and peristalsis in early postoperative period.

Cause-and-effect relation of adhesion process and hypoxia were described in a number of publications [9], but there is still no quantitative estimate of these processes association. Particularly, A.H.Haken (2001) noted, that supramedian laparotomy decreases vital capacity of lungs more considerably than thoracotomy, provoking so cellular hypoxia. Postoperative paresis of gastrointestinal tract after liquidation of bowel obstruction cause intestinal failure syndrome in $40-96 \%$ cases [10].

R.A.Zhenchevskiy (1989) proved that it is not necessary to peritonize peritoneum defect, because mesothelium is implanted from unimpaired edge regions, and suturing lead to tissue ischemia and fibrosis development [6].

Adhesive process in patients after peritonitis concentrates in lower parts of abdominal cavity, that is related with pathological exudate concentration exactly in the region of small pelvis and duration of its exposure. Adhesion process begins after 2 hours from the moment of mesothelium damage. Especially it is observed in course of prolong surgical interventions, when already separated intestinal loops begin to adhere. In course of 24 hours in the abdominal cavity protein fraction exudation take place, fibroblast proliferation, fibrin formation, which a coarse-fiber connective [11;12].

Presence of postoperative cicatrix and periodically arising pains in stomach are interpreted by surgeons and gastroenterologists as a peritoneal commissures with assigning the same type of treating. Therein patients attitude towards their condition decrease, that leads to their late appeal for medical assistance. 
Exists an opinion concerning ineffectiveness and prospectless of abdominal cavity peritoneal commissures planned operative treatment. A number of surgeons consider that each new operation only worsens patient's condition. Skeptical attitude of surgeons to planned adhesiolysis promote adhesive process propagation and necessitates surgical treatment at already developed complications, that undoubtedly increases risk of mortality [5].

Unsolved remained the question why some commissures cause painful sensation while the others remain clinically inert? From here a practical dilemma arises - which adhesions should be separated? To the mind of a number of authors, pain in stomach is a valid symptom of adhesion process and continuous chronic pain witness about involvement of mesoceccum.

Sensation of pain causes tension of visceral and parietal peritoneum, and also absence of internal organs physiological displaceability. Therewith in patients with complete abdominal cavity obliteration pain sensation is localized in a certain region and may not relate to an obstacle. Abdominal pain is first of all determined by tension of entodermal canal in adductive, not being involeved in adhesive process, intestine $[2 ; 12]$.

Operating surgeon has to select: is it necessary to operate a patient with bowel obstruction, with frequent pain attacks? Is it reasonable to transect symptomless adhesions? What volume of adhesiolysis is optimal one? What is the sequence of operation, one of the stages of it would be adhesiolysis? Is it possible to attain quality of life improvement using conservative treatment?

To the opinion of a number of authors, adhesiolysis should be used only when complications in the main stage of operation arise or in the case of adhesive intestinal obstruction for obstacle removing. Another researches inclines to complete adhesion lysis, considering that revision of abdominal organs with adhesives separation in necessary for any pathology, including hernioplasty, if operative access permit its performance $[3 ; 5]$.

According to modern notions, partial adhesiolysis performance is more justified and provides good long-term results in $55 \%$ cases, as opposed to total separation of interintestinal adhesion, in which satisfactory results are attained only in $28 \%$ cases $[14 ; 15]$.

To our mind, at total adhesive process spreading, complicated by adhesive obstruction, it is not necessary to separate all the adhesions in abdominal cavity; it is sufficient to restrict it only by obstacles removing. But it is necessary to pay attention that in distal region of collapsed intestine pathological formations may also presented (strictures, band adhesions, intestine torsions), which may become obstacle while straightening adducting region. Thus "surgical treatment" of patients is not finished after surgical intervention, but continues till full intestinal transport restoration [16].

Surgical interventions performed by laparatomy access, to the opinion of a number of authors, lead to abdominal adhesions formation in $60-90 \%$ cases. It was proved that: the most efficient methods of intestinal obstruction prevention and treating are low traumatic surgical interventions [17].

Laparoscopic adhesion lysis is a primary treatment method of peritoneal commissures [18]. Low invasive methods application does not guarantee complete deliverance from adhesions [8]. Until quite recently presence in anamnesis abdominal incision was contraindication to laparoscopic intervention [19]. In modern surgical clinic laparoscopic adhesiolysis is an operation of choice in case of abdominal cavity peritoneal commissures and at acute adhesive bowel obstruction as well. 
Introduction into day-to-day surgeons activity laparoscopic techniques permited to widen surgical intervention spectrum at abdominal cavity organs. Laparoscopic technique is more often used in treatment of peritoneal commissures and its complications. But in separating vast visceroparietal adhesions with application of arbitrary methods wound surfaces are formed, often leading to commissures recidivism.

To complications of laparoscopic adhesiolysis heat injury should be referred. For adhesiotomy in majority cases diathermo-coagulation is applied, which often lead to burn of intestinal wall $[11 ; 20]$. These injuries are left unnoticed and revealed in $7-10 \%$ cases by intestinal perforation, $[1 ; 21]$. Application of unipolar coagulation even more dangerous due to nearest organs and tissues traumatizing. The most preferable are ultrasonic techniques of decollement.

Meta-analysis, performed by a group of researchers proved the absence of statistically significant difference between open and laparoscopical adhesiolysis in a number of intraoperative bowel damages, wound infections and mortality. Vice versa, statistically significant difference in the frequency of general and pulmonary complications at laparoscopic operation as compared with open ones was observed. The authors come to the conclusion that laparoscopic access is safer in case of sufficiently experienced surgeons $[3 ; 22]$.

Laparoscopic adhesiolysis at expanded intestine loops and adhesive process of III-IV stages increases risk of paratherapeutic injuries of intestine [23;24].

It is necessary to refuse from laparoscopic adhesiolysis if prolong lymphatic visceroparietal adhesions at hollow organ were detected. Therewith, in early postoperative period it is reasonable to resort to programmable sanitations of abdominal cavity, especially for patients with signs of peritonitis or with prerequisites to its appearance.

Frequency of intestine injuries at laparoscopic adhesiolysis in conditions of intestinal obstruction, according to the data provided by a number of authors, constitutes from 6.3 to $26.9 \%[7 ; 9 ; 10]$.

Contraindication to laparoscopic adhesiolysis application for the last decade rapidly decreased. The most appropriate and undeniable contraindications at the moment are:

- cardiovascular diseases in decompensation stage;

- respiratory embarrassment in decompensation stage;

- final stages of pregnancy( $3^{\text {rd }}$ trimester $)$;

- radiotherapeutics at pathology of abdominal cavity organs radiotherapeutics in anamnesis;

- over two operations for abdominal cavity peritoneal commissures;

- giant ventral hernia;

- presence of intestinal stoma.

Predictors of successful laparoscopic treatment of acute adhesive intestinal obstruction are the following: no more than 2 laparotomy in anamnesis, one of which - appendectomy, absence of previous middle laparotomy incision and singular adhesions [15]. A special group consists of patients, who earlier received radiotherapy at pathology of abdominal cavity organs. Performance of laparoscopic adhesiolysis present in such patients significant difficulties [16]. 
Important question is to determine indications for decompression and intestine intubation, benefit or harm of which directly depends on meeting the following requirements: Probe evenly conducted at all the length of intestine, loops of small intestine are laid down with three bends, simultaneous implementation of intestine content aspiration. Sometimes probe behavior itself inflict a greater damage to intestinal wall, that blood micro-circulation disorder or hypertension in intraintestine. Intramucosal intestine layer destructs, appeared hematomas, bedsores, ruptures of muscular layer, probe is not conducted to ileocaecal angle. All these circumstances often tranform intestine intubation from useful therapeutic procedure into traumatic one, worsening small intestine wall condition and patient himself.

Sewing deserosations contribute adhesions formation, increase ischemia and provoking necrosis development. Besides, growth of adhesions stimulate application of suture material and squeezing tissues in seam. Modern literature contains sufficient justification for canceling from zones deserosations closing. From the other side, presence of vast wound surfaces considerably increase the level of tissues factors of fibrogenesis. Deep deserosation in conditions of intestinal paresis, increase of intra-abdominal and intra-intestinal pressure, and also blood circulation disorder in intestinal wall, considerably increase probability of stress ulcers formation with intestinal perforation in earlier postoperative period.

Conner stone in the battle with peritoneal commissures of abdominal cavity is prevention of adhesions formation. At that moment there are empirically defined preventive measures with low efficiency. Extremely complex task is to predict recidivisms probability, which may reach $60-70 \%$, wherein each previous episode complicates the following, and repeated compelled resections make entodermal canal shorter $[3 ; 11]$.

Surgical methods of prevention adhesion formation are numerous. For example, enteropexy, at which for prevention of adhesive bowel obstruction in case of expressed adhesions inside abdominal cavity during repeated operations small bowel loops lay down and fix in the form of vertically located battery by means of application continuous seromuscular suture from mesenteric root to bending of small bowel [25]. As supposed, that method enables to provide prevention measures against appearance of adhesive bowel obstruction. It is important to avoid formation of single conglomerate of small bowel loops, excluding ability of repeated reconstructive operations; disorder of bowel blood supply with infections activation; development of refractory paresis in gastrointestinal tract, and thereby to attain more reliable preventive measures in respect of adhesive intestinal obstruction arise, appearance of adhesive bowel obstruction.

But these methods have a number of disadvantages: formation of loops conglomerate of small intestine with involvement in it the whole mesentery make impossible performance of repeated reconstructive operations, hereby condemning patient on inevitable death in case of acute intestinal obstruction recidivism; suturing at presence of initial changes of mesentery blood circulation and chronic mesenteric adenitis critically disorder blood supply of small intestine wall and stipulate microflora activation; loops fixation acutely suppress peristalsis and lead to postoperative enteroparesis.

Thus, approaches to diagnostics, treatment and prevention of abdominal cavity peritoneal commissures have a number of contradictions and require further study. 


\section{Conclusions}

The most efficient methods of intestinal obstruction prevention and treating are low traumatic surgical interventions.

Tissue hypoxia and inflammation affect fibroblast proliferation and connective tissue maturation. Minimization of tissue hypoxia during surgery and in the early postoperative period is one of the methods for the prevention of adhesions and requires further study.

The absence of statistically significant difference between open and laparoscopical adhesiolysis in a number of intraoperative bowel damages, wound infections and mortality was proved. Laparoscopic operations if compared with open ones have less number of general and pulmonary complications.

\section{References}

1. Hackenberg T., Mentula P., Leppaniemi A., Sallinen V. Laparoscopic versus open surgery for acute adhesive small-bowel obstruction: a propensity score-matched analysis. Scand. J. Surg., 2017, no. 106 (1), pp. 28-33.

2. Baghdadi Y.M.K., Morris D. S., Choudhry A. J., Thiels C. A., Khasawneh M. A., Polites S. F., Goussous N., Jenkins D. H., Zielinski M.D. Validation of the anatomic severity score developed by the American Association for the Surgery of Trauma in small bowel obstruction. J. Surg. Res., 2016, no. 204 (2), pp. 428-434.

3. Strik C., Stommel M. W., Schipper L. J., Van G.H., Ten Broek R.P. Risk factors for future repeat abdominal surgery. Langenbeck's Arch, Surg., 2016, no. 401 (6), pp. 829-837.

4. Zilber A.P. Clinical physiology in anesthesiology and resuscitation. Moscow, Meditsina Publ., 1984. 479 p. (In Russian)

5. Loftus T., Moore F., VanZant E., Bala T., Brakenridge S., Croft C., Lottenberg L., Richards W., Mozingo D., Atteberry L., Mohr A., Jordan J. A protocol for the management of adhesive small bowel obstruction. J. Trauma Acute Care Surg., 2015, no. 78 (1), pp. 13-19.

6. Zhenchevskiy R. A. Adhesive disease. Moscow, Medicina Publ., 1989. 192 p. (In Russian)

7. Grafen F.C., Neuhaus V., Schob O., Turina M. Management of acute small bowel obstruction from intestinal adhesions: indications for laparoscopic surgery in a community teaching hospital. Langenbeck's Arch. Surg., 2010, no. 395 (1), pp. 57-63.

8. Ray N. F., Denton W. G., Thamer M., Henderson S. C., Perry S. Abdominal adhesiolysis: inpatient care and expenditures in the United States in 1994. J. Am. Coll. Surg., 1998, no. 186, pp. 1-9.

9. Wullstein C., Gross E. Laparoscopic compared with conventional treatment of acute adhesive small bowel obstruction. Br. J. Surg., 2003, no. 90 (9), pp. 1147-1151.

10. Galperin Yu. M. Paresis, paralysis and functional intestinal obstruction. Moscow, Medicina Publ., 1975. 219 p. (In Russian)

11. Vorobiev A. A. Prevention of adhesions of the abdominal cavity (review of foreign literature). Khirurgiya, 1998, no. 3, pp. 65-68. (In Russian)

12. Jeppesen M., Tolstrup M. B., Gogenur I. Chronic pain, quality of life, and functional impairment after surgery due to small bowel obstruction. World J. Surg., 2016, no. 40 (9), pp. 2091-2097.

13. Ten Broek R. P., Bakkum E. A., Laarhoven C. J., van Goor H. Epidemiology and prevention of postsurgical adhesions revisited. Ann Surg., 2016, no. 263 (1), pp. 12-19.

14. Johnson K. N., Chapital A. B., Harold K. L., Merritt M. V., Johnson D. J. Laparoscopic management of acute small bowel obstruction: evaluating the need for resection. J. Trauma Acute Care Surg., 2012, no. 72 (1), pp. 25-30.

15. Farinella E., Cirocchi R., La M. F., Morelli U., Cattorini L., Delmonaco P., Migliaccio C., De Sol A. A., Cozzaglio L., Sciannameo F. Feasibility of laparoscopy for small bowel obstruction. World J.Emerg. Surg., 2009, no. 4:3, pp. 27-45.

16. Nakamura T., Ishii Y., Tsutsui A., Kaneda M., Sato T., Watanabe M. Safety and indications of laparoscopic surgery for postoperative small-bowel obstruction: a single-center study of 121 patients. Surg. Laparosc. Endosc. Percutan. Tech., 2017, no. 27 (4), pp. 301-305.

17. Wu Z., Boersema G.S., Dereci A., Menon A. G., Jeekel J., Lange J.F. Clinical endpoint, early detection, and differential diagnosis of postoperative ileus: a systematic review of the literature. EurSurg Res., 2014, no. 3-4, pp. 127-138. 
18. Dronov A. F. Laparoscopic operations for acute adhesive small bowel obstruction (literature review). Endoskopivheskaya khirurgiya, 2000, no. 6, pp. 43-48. (In Russian)

19. Nifantiev O.E. Mechanical obstruction of the duodenum. Krasnoyarsk: Izdatelstvo Krasnoyakskogo universiteta Publ., 1984. 97 p. (In Russian)

20. Behman R., Nathens A. B., Byrne J. P., Mason S., Look H. N., Karanicolas P. J. Laparoscopic surgery for adhesive small bowel obstruction is associated with a higher risk of bowel injury: a population-based analysis of 8584 patients. Ann. Surg., 2017, no. 266 (3), pp. 489-498.

21. Maringof A.L. A new approach to the prevention and treatment of postoperative adhesions. Endoskopivheskaya khirurgiya, 2007, no. 6, pp. 34-35. (In Russian)

22. Li M.Z., Lian L., Xiao L.B., Wu W.H., He Y.L., Song X.M. Laparoscopic versus open adhesiolysis in patients with adhesive small bowel obstruction: a systematic review and meta-analysis. Am. J. Surg., 2012, no. 204, pp. 779-786.

23. Strik C., Stommel M. W., Ten Broek R. P., Van G. H. Adhesiolysis in patients undergoing a repeat median laparotomy. Dis. Colon. Rectum., 2015, no. 58 (8), pp. 792-798.

24. Ten Broek R.P., Strik C., Van G. H. Preoperative nomogram to predict risk of bowel injury during adhesiolysis. Br. J. Surg., 2014, no. 101 (6), pp. 720-727.

25. Noble T. B. J. Intem. Coll. Surg., 1958, no. 31 (3), p. 286.

Received: January 15, 2021

Accepted: March 25, 2021

Authors'information:

Dmitry A.Kluiko — PhD, Associate Professor; mdkluiko@gmail.com

Vladimir E. Korik - Dr. Sci. in Medicine, Professor; voet@tut.by

Alexey N.Sivets — PhD; sivets76@mail.ru 